\title{
Radioimmunotherapy in Non-Hodgkin Lymphoma: Opinions of U.S. Medical Oncologists and Hematologists
}

\author{
Niklaus G. Schaefer ${ }^{1}$, Jiemin $\mathrm{Ma}^{2}$, Peng Huang ${ }^{3}$, Judy Buchanan ${ }^{1}$, and Richard L. Wahl ${ }^{1}$ \\ ${ }^{1}$ Division of Nuclear Medicine, Russell H. Morgan Department of Radiology and Radiological Sciences, Johns Hopkins University \\ School of Medicine, Baltimore, Maryland; ${ }^{2}$ Department of Epidemiology, Johns Hopkins University, Baltimore, Maryland; and \\ ${ }^{3}$ Division of Oncology Biostatistics, Johns Hopkins University, Baltimore, Maryland
}

\begin{abstract}
Radioimmunotherapy is approved by the Food and Drug Administration for CD20 antigen-positive follicular and transformed non-Hodgkin lymphoma. The goal of this study was to obtain the opinion of hematologists and medical oncologists about CD20-directed radioimmunotherapy in the United States. Methods: An e-mail-based survey with 8 questions was sent to 4,239 oncologists and hematologists throughout the United States. Results: Two hundred sixteen (5.0\%) oncologists and hematologists responded to our survey. One hundred fifty-seven $(72.7 \%)$ said they had referred patients with non-Hodgkin lymphoma for radioimmunotherapy in the last 24 mo. Different types
\end{abstract} of practices had significantly different concerns regarding this treatment. Compared with referring physicians from academic centers, those from nonacademic centers reported significantly higher concerns about the lack of a site to which to refer patients for treatment $(P<0.01)$, the lack of interest by nuclear physicians in this type of treatment $(P<0.01)$, and a referral process that they felt was too complicated $(P<0.01)$. They were also more concerned about an economically adverse effect on their own practices if they referred patients for radioimmunotherapy $(P<$ 0.01 ). Referring physicians who perceived consolidation as a possible indication for radioimmunotherapy had significantly fewer concerns about an adverse effect on their own practice $(P<0.01)$ and about nonradioactive alternatives $(P<0.01)$. Seventy-nine $(36.6 \%)$ responders thought radioimmunotherapy would probably grow in importance, and 52 (24.1\%) responders thought it would definitely grow in importance. However, the group with a positive outlook about the future of radioimmunotherapy predicted a higher growth of radioimmunotherapy if they could administer it in their own offices $(P<0.05)$. Conclusion: Radioimmunotherapy was generally viewed positively by referring physicians. However, in addition to scientific concerns, barriers to the use of radioimmunotherapy included difficulty in referral, perceptions of a high cost of the treatment, concerns about negative financial outcomes related to referral, and an opinion that the treatment would be used more if given by medical oncologists in their own offices. For the growth of radioimmunotherapy, it appears crucial not only to demonstrate the treatment's safety and efficacy but also to streamline the

Received Nov. 25, 2009; revision accepted Feb. 3, 2010.

For correspondence or reprints contact: Richard L. Wahl, Division of Nuclear Medicine, Russell H. Morgan Department of Radiology and Radiological Sciences, Johns Hopkins University School of Medicine,

Room 3223, JHOC 601, N. Caroline St., Baltimore, MD 21287-0817. E-mail: rwahl@jhmi.edu

COPYRIGHT ( 2010 by the Society of Nuclear Medicine, Inc. referral process, to enhance collaboration between specialists, and-it appears - to develop economic incentives for the referring physician.

Key Words: lymphoma; radioimmunotherapy; survey; pharmacoeconomics

J Nucl Med 2010; 51:987-994

DOI: 10.2967/jnumed.109.073155

$\mathbf{T}$ he radiolabeled monoclonal antibodies ${ }^{90}$ Y-ibritumomab tiuxetan (Zevalin; Spectrum Pharmaceuticals) and ${ }^{131}$ I-tositumomab (Bexxar; GlaxoSmithKline) are approved for the treatment of patients with follicular and transformed non-Hodgkin lymphoma (NHL) who failed to respond to or relapsed after prior therapies, including rituximab (Rituxan; Biogen Idec/Genentech) and standard chemotherapy. Recently, ${ }^{90}$ Y-ibritumomab tiuxetan was approved by the Food and Drug Administration (FDA) as a part of the initial therapy of follicular NHL in the United States.

Proof of concept of ${ }^{131}$ I bound to anti-CD20 antibodies in patients with relapsed CD20-positive follicular lymphoma (FL) was published in the early 1990s (1). These initial exciting data were confirmed in a phase II trial by the same group and in multicenter studies. ${ }^{131} \mathrm{I}$ anti-CD20 antibodies produced a high overall response rate, and approximately one third of patients had a complete remission despite having chemotherapy-refractory low-grade or transformed low-grade NHL or having experienced a relapse earlier after chemotherapy (2). In untreated patients with advanced-stage FL, ${ }^{131}$ I-tositumomab showed an overall response rate of $95 \%$, with a rate of complete remission of $74 \%$. In this study, the 5-y progression-free survival was $59 \%$, the toxicity was moderate myelosuppression, and no cases of myelodysplastic syndrome (MDS) or acute leukemia had been observed at the time of the report (3).

Several studies have now reported the benefit of ${ }^{90}$ Y-ibritumomab tiuxetan in relapsed or refractory CD20-positive FL (4-6). A recent large, prospective phase 
III trial investigated ${ }^{90} \mathrm{Y}$-ibritumomab tiuxetan in the setting of consolidation of first-line therapy and showed high efficacy with no unexpected toxicities (7). This study led to the approval of ${ }^{90}$ Y-ibritumomab tiuxetan for consolidation treatment as a part of first-line therapy in both Europe and now the United States. Other anti-CD20 radiopharmaceuticals, including ${ }^{131}$ I-rituximab, have shown benefit in relapsed or refractory indolent NHL, with a high overall response rate of $76 \%(8)$.

Despite these reports of efficacy and the reasonable safety of ${ }^{90} \mathrm{Y}$-ibritumomab tiuxetan and ${ }^{131}$ I-tositumomab, as well as FDA and European Medicines Agency (EMEA) approvals, these treatments appear to be used much less frequently than alternative nonradioactive treatments. The reasons for this low use of $\mathrm{CD} 20$-directed radioimmunotherapies are unclear. A front-page article in The New York Times (9) discussed innovative treatments, market factors, and health politics in the choices of treatment for NHL. This article claimed that in the United States the options for drugs to treat cancer, such as radioimmunotherapy, are limited by market forces (9). A further reason for possible underuse was believed to be related to relatively low reimbursement (of the cost of both the drug and the treating physicians) for the administration of these radioimmunotherapeutics (10). Other concerns included the wide range of nonradioactive alternative treatments for CD20-positive lymphomas, possible unexpected late side effects of radioimmunotherapy, and a complicated patient-referral process. Few objective data are available, however, on how oncologists choose to use, or not to use, radioimmunotherapy in patients with NHL.

The goal of this study was to survey referring oncologists for their opinion about CD20-directed radioimmunotherapy in the United States.

\section{MATERIALS AND METHODS}

We developed an 8-question survey (Fig. 1), and after testing it in a pilot setting at our own center, used it to systematically question medical oncologists and hematologists about their perception of radioimmunotherapy in NHL. The survey assessed the process of deciding on whether to treat NHL patients with radioimmunotherapy. General perceptions regarding the treatment in the broad areas of evidence-based efficacy, alternative therapeutic options, and logistic barriers to use were explored. Questions about practice-specific and societal economic factorsparticularly emphasizing possible concerns about this form of therapy that might detract from its use-were included. To increase the response rate, the questions were kept brief so that the survey could be completed within $10 \mathrm{~min}$.

SurveyGizmo (Widgix, LLC), an e-mail-based tool drawing on the databases of medical oncologists and hematologists, was used to distribute the survey. Most of the questions were multiplechoice; some had a free-text option. One question about a PET facility in the oncologists' or hematologists' office was not used for this survey and will be reported elsewhere. The Institutional Review Board of the Johns Hopkins University approved the study protocol. Potential responders were informed of the principal investigator's name but were not told whether the principal investigator had any financial interest in either of these forms of therapy. We calculated means and SD. For the multivariate analyses, logistic regression or proportional odds models were fitted for binary or ordered categoric response variables, and linear regression models were fitted for physician concern variables. These models will adjust for potential confounding covariates such as geographics and practice type.

\section{RESULTS}

Demographic Characteristics of Study Participants

We e-mailed 4,239 surveys. The names and addresses of the recipients were culled from a combination of databases, and we received $216(5.0 \%)$ completed responses. The survey was resent twice at intervals of $7 \mathrm{~d}$ if there was no response and was closed after $31 \mathrm{~d}$. A map of the geographic location of the responders is provided in Figure 2. Responders specified their predominant practice location: $82(38 \%)$ worked in university hospitals, $21(9.7 \%)$ in nonuniversity hospitals, $9(4.2 \%)$ in private multispecialty offices, $99(45.8 \%)$ in private oncology offices, and 5 $(2.3 \%)$ in industry. Our survey allowed only 1 selection for the major type of practice (Fig. 3).

\section{Referral Frequencies and Indications}

Twenty-one $(9.7 \%)$ responders saw radioimmunotherapy with ${ }^{131}$ I-tositumomab or ${ }^{90} \mathrm{Y}$-ibritumomab tiuxetan as a possible first-line indication, and 111 (51.4\%) would refer NHL patients for second-line treatment. One hundred thirty-eight $(63.9 \%)$ saw this treatment as third-line treatment, and $71(32.9 \%)$ would use it later than third-line treatment. Nine $(4.2 \%)$ thought radioimmunotherapy with ${ }^{131}$ I-tositumomab or ${ }^{90}$ Y-ibritumomab tiuxetan was only investigational at this time. Three $(1.38 \%, 2$ from academia and 1 from a nonacademic hospital) stated they would never refer a patient with NHL for radioimmunotherapy. Fifty-four (25\%) stated they would refer patients for consolidation after immunochemotherapy. This survey was completed just before U.S. FDA approval of ${ }^{90}$ Y-ibritumomab tiuxetan as a part of first-line treatment. Figure 4 reports the physician's perceived indications for ${ }^{131}$ I-tositumomab or ${ }^{90} \mathrm{Y}$-ibritumomab tiuxetan in patients with NHL.

\section{Concerns About Anti-CD20 Radioimmunotherapy}

The surveyed population rated their concerns about radioimmunotherapy for NHL from 1 (no concern) to 5 (major concern). Nine factors were rated. In decreasing order of concern, these were as follows: (1) ${ }^{131}$ I-tositumomab or ${ }^{90}$ Y-ibritumomab tiuxetan theoretically may cause bone marrow damage preventing further therapy (autologous stem cell transplantation) (mean rating, $3.16 \pm 0.09$ ). (2) There might be unexpected late side effects of ${ }^{131}$ I-tositumomab or ${ }^{90}$ Y-ibritumomab tiuxetan (MDS) (mean rating, $3.04 \pm 0.08$ ). (3) There are too many effective nonradioactive treatment alternatives for $\mathrm{NHL}$ (mean rating, $3.0 \pm 0.09$ ). (4) ${ }^{131}$ I-tositumomab or 


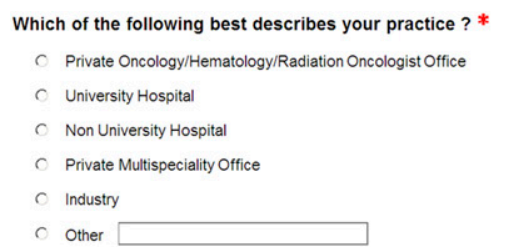

Do you refer patients with Non Hodgkins Lymphoma for Radioimmunotherapy with Bexxar or Zevalin?

C No

C. Yes, $1-3$ in the last 24 months

C. Yes, $4-7$ in the last 24 months

Yes, $8-14$ in the last 24 months

Yes, $>15$ in the last 24 months

Non Hodgkins Lymphoma treatment with Bexxar or Zevalin: When should it be used ? (multiple options possible)

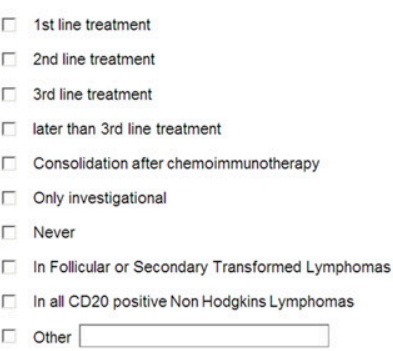

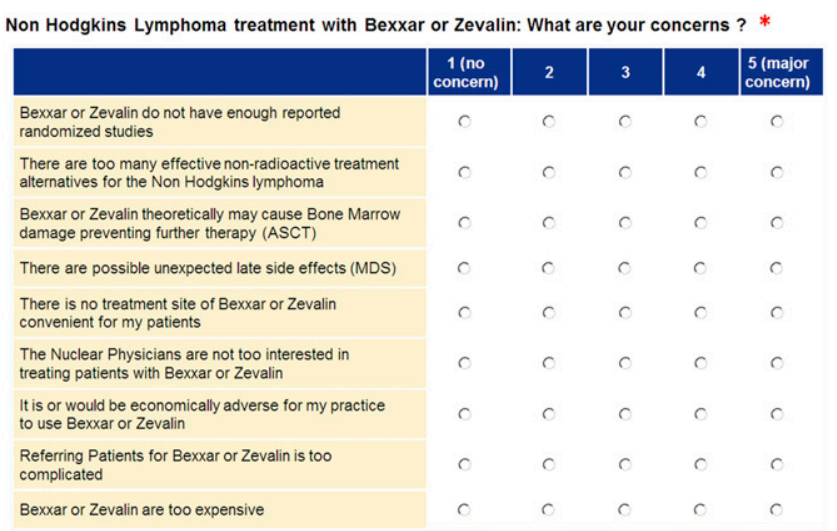

If Radiation Regulations should allow treatment with Bexxar and Zevalin in your own office: Would Bexxar and Zevalin grow in importance?

$\circ$ Yes

( No


FIGURE 1. The survey (square boxes represent multiple answers possible; circles represent only 1 answer possible; items marked with * are required).

${ }^{90}$ Y-ibritumomab tiuxetan treatment is too expensive (mean rating, $2.96 \pm 0.10$ ). (5) Referring patients for ${ }^{131}$ I-tositumomab or ${ }^{90} \mathrm{Y}$-ibritumomab tiuxetan is too complicated (mean rating, $2.50 \pm 0.10$ ). (6) Nuclear physicians are not too interested in treating patients with ${ }^{131} \mathrm{I}$-tositumomab or ${ }^{90}$ Y-ibritumomab tiuxetan (mean rating, $2.44 \pm$ 0.10). (7) ${ }^{131}$ I-tositumomab or ${ }^{90}$ Y-ibritumomab tiuxetan treatment does not have enough reported randomized studies (mean rating, $2.38 \pm 0.09$ ). (8) There is no treatment site of ${ }^{131}$ I-tositumomab or ${ }^{90} \mathrm{Y}$-ibritumomab tiuxetan that is convenient for my patients (mean rating, $2.12 \pm 0.10$ ). (9) It is or would be economically adverse for my practice to use ${ }^{131}$ I-tositumomab or ${ }^{90} \mathrm{Y}$-ibritumomab tiuxetan (mean rating, $2.05 \pm 0.09)$.

\section{Predicted Growth of Radioimmunotherapy for NHL}

Ten $(4.62 \%)$ responders thought radioimmunotherapy for NHL would definitely not grow in the future, $27(12.5 \%)$ thought it would probably not grow, 46 (21.3\%) were uncertain about the future growth, 79 (36.5\%) thought it would probably grow, and $52(24.1 \%)$ thought it would definitely grow (Fig. 5).

\section{Predicted Growth of Approved Radioimmunotherapy in Responder's Own Office}

Ninety-three (43.1\%) responders felt that radioimmunotherapy with ${ }^{131}$ I-tositumomab or ${ }^{90} \mathrm{Y}$-ibritumomab tiuxetan would grow in importance if they could give it in their own practice. One hundred twenty-three $(56.9 \%)$ responders felt this would not affect use of the treatment in their practice.

\section{Further Comments from Responders}

This free-text field was open for further comments. Forty-six $(21.3 \%)$ responders added one or more specific comments. The comments could be categorized easily as medical, scientific, logistic, or economic. Nine comments raised medical concerns: the referring physicians were concerned about possible prolonged myelosuppression and MDS after radioimmunotherapy. Eleven comments pointed toward a lack of randomized trials comparing 


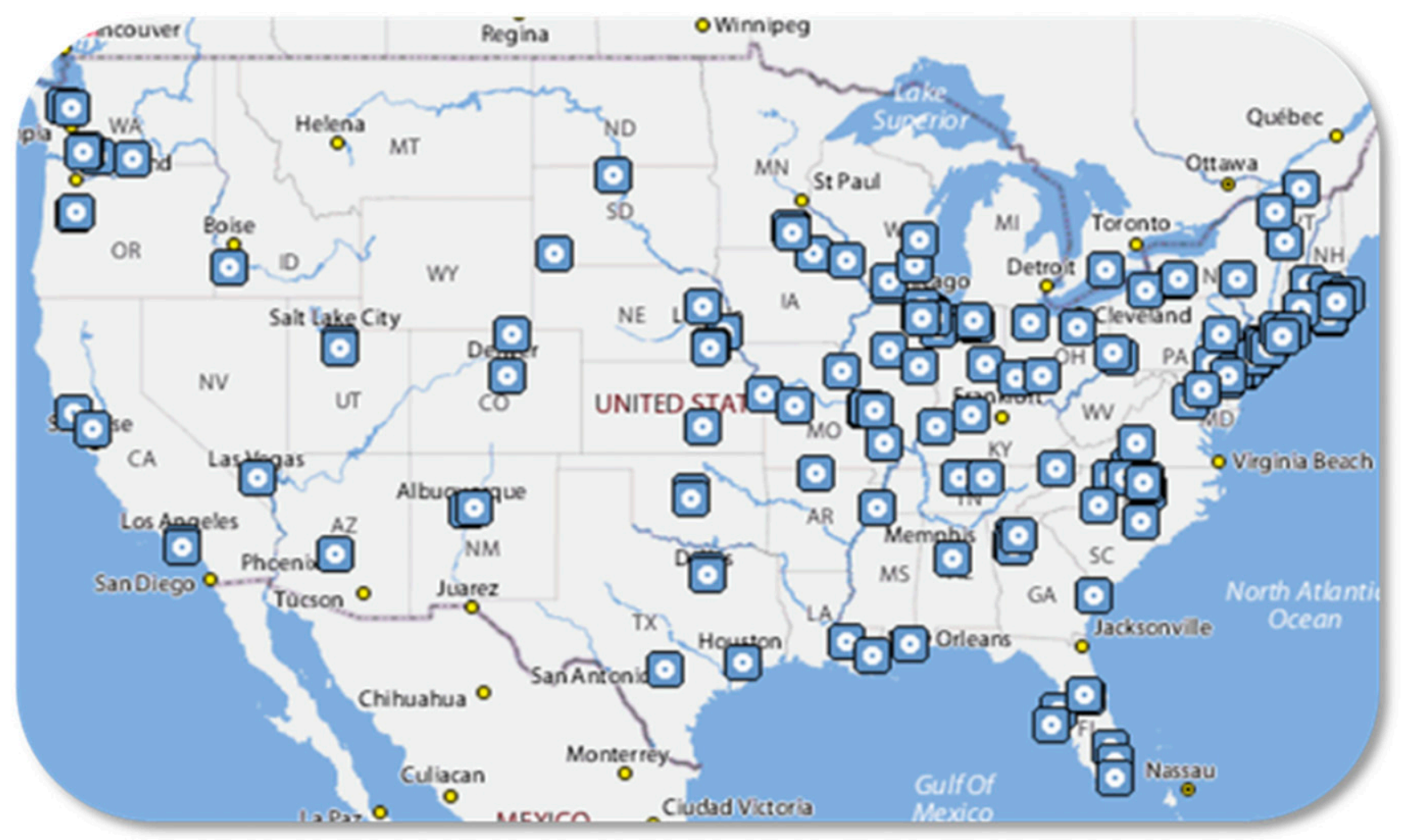

FIGURE 2. Geographic locations of responders (Yahoo! MapMaker for Excel, version 1.02; Microsoft).

radioimmunotherapy with conventional treatment. Logistic concerns were raised in 13 comments. Several comments specifically pointed out the complicated referral process and their nuclear physicians' lack of interest in using radioimmunotherapy. Further logistic concerns included losing the patient after referral to an academic medical institution. Thirteen responders expressed economic concerns. They reported the possibility that their practice would not be reimbursed because their patients were referred to another site for radioimmunotherapy. They also

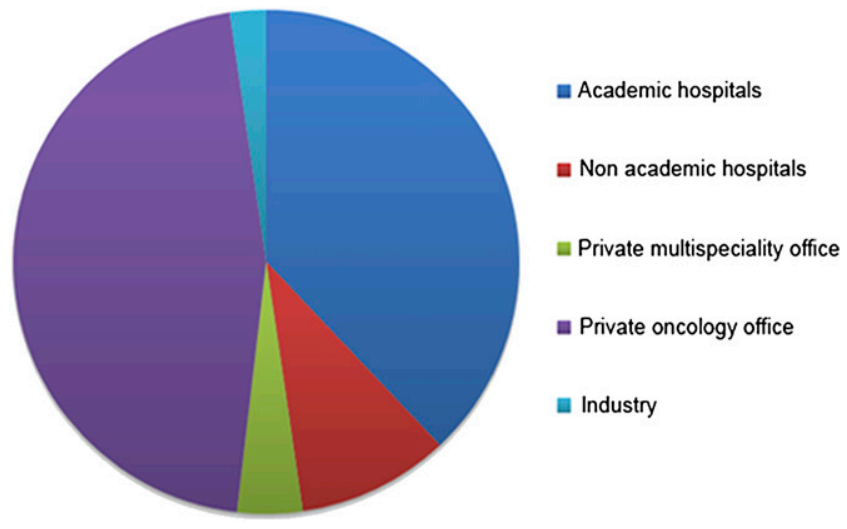

FIGURE 3. Practice types: academic hospitals, 38\%; nonacademic hospitals, 9.7\%; private multispecialty offices, $4.2 \%$; private oncology offices, $45.8 \%$; and industry, $2.3 \%$. agreed that if regulations allowed them to administer ${ }^{90}$ Y-ibritumomab tiuxetan or ${ }^{131}$ I-tositumomab within their own practice, their use of this therapy would increase. Further concerns were expressed about reduced reimbursement for standard treatments if the oncologists were to send patients out of their practices for radioimmunotherapy.

\section{Emerging Patterns After Subgroup Analysis}

Referral Frequency, by Type of Practice. There was no significant difference in practice type among responders

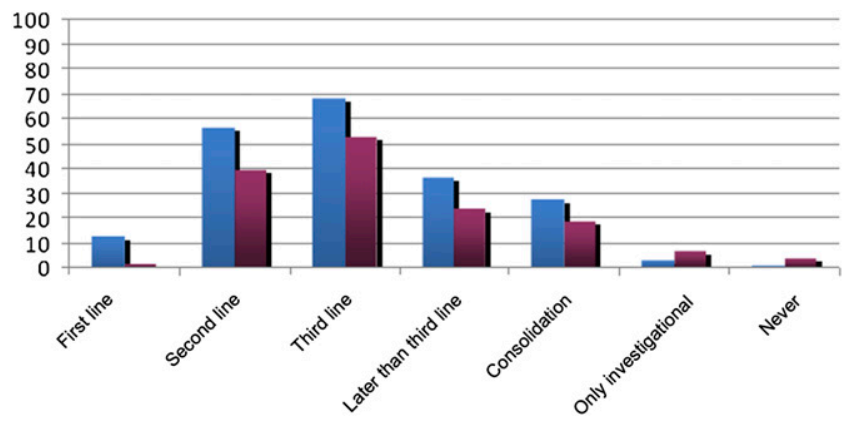

FIGURE 4. Preferred indications (\%) for use of ${ }^{131}$ Itositumomab or ${ }^{90}$-ibritumomab tiuxetan. Blue = physicians who did treat $\mathrm{NHL}$ patients with ${ }^{131}$ I-tositumomab or 90Y-ibritumomab tiuxetan in last $24 \mathrm{mo}(n=157)$; red = physicians who did not $(n=59)$. 


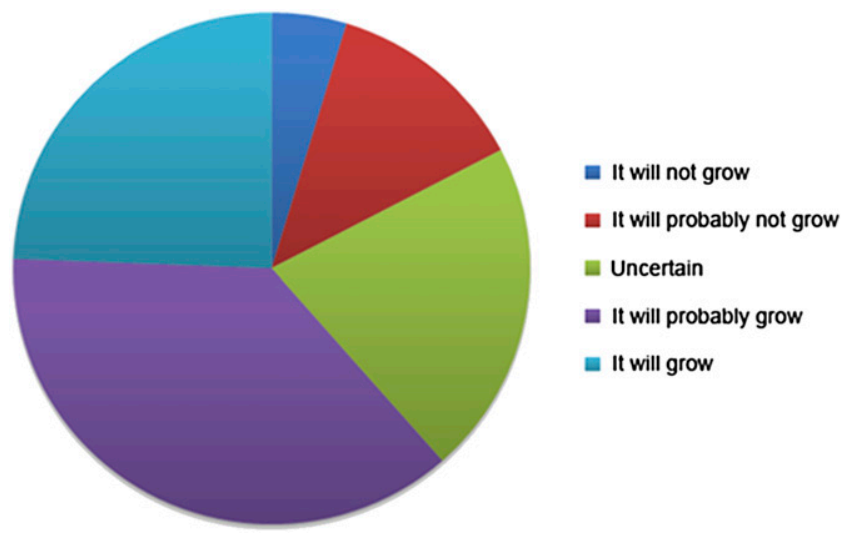

FIGURE 5. Predicted future growth of ${ }^{131}$-tositumomab or ${ }^{90}$ Y-ibritumomab tiuxetan radioimmunotherapy for $\mathrm{NHL}$ : It will not grow, $4.62 \%$; it will probably not grow, $12.5 \%$; uncertain, $21.3 \%$; it will probably grow, $35.6 \%$; and it will grow, $24.1 \%$.

who had referred a patient for radioimmunotherapy (academia vs. nonacademia, $P=0.78$; hospitals vs. private practice, $P=0.43$ ). However, the referral frequency was significantly higher in hospitals than in private oncology or multispecialty practices $(P<0.01)$.

Referral Indication, by Type of Practice. No significant difference between 1 particular line of treatment and a responder's type of practice was observed. Also, responders who saw consolidation as a possible indication after chemoimmunotherapy for their patients with NHL did not significantly differ in their type of practice.

Concerns About Anti-CD20 Radioimmunotherapy. Responders not in academia were more concerned than those in academia about access to a treatment site $(P<0.01)$, lack of interest by nuclear physicians in these treatments
$(P<0.01)$, a referral process that is too complicated $(P<$ $0.01)$, and the economic adversity of referring patients for radioimmunotherapy $(P<0.01)$ (Table 1$)$.

Responders from private practices had more concerns than did responders from hospitals about possible MDS $(P<0.1)$ after radioimmunotherapy, bone marrow damage preventing further therapy $(P<0.05)$, lack of a treatment site for patients with NHL $(P<0.01)$, lack of interest by nuclear physicians $(P<0.1)$, and a referral process that was too complicated $(P<0.01)$.

The only significant concern of the subgroup who had referred patients in the last 24 mo was the perception that the higher expense of ${ }^{90} \mathrm{Y}$-ibritumomab tiuxetan and ${ }^{131} \mathrm{I}$-tositumomab is associated with lower use $(P<0.05)$.

Responders who would not refer NHL patients for firstor second-line treatment with ${ }^{131} \mathrm{I}$-tositumomab or ${ }^{90}$ Y-ibritumomab tiuxetan tended to believe other nonradioactive treatment alternatives were available $(P<0.1)$. Physicians favoring early-line treatment did not significantly differ from other physicians in medical or scientific, logistic, or economic concerns. Responders who viewed consolidation as a less appropriate indication for radioimmunotherapy had significantly more concerns about nonradioactive treatment alternatives $(P=0.02)$, bone marrow damage preventing further therapy $(P=0.02)$, and the occurrence of later MDS $(P=0.05)$. However, the most significant concern in this group, which did not consider consolidation to be an indication for radioimmunotherapy, was a perceived adverse economic effect on their own practice from the use of radioimmunotherapy for consolidation $(P<0.01)$.

Oncologists who predicted an increase in the importance of ${ }^{131}$ I-tositumomab or ${ }^{90} \mathrm{Y}$-ibritumomab tiuxetan use in their own office, should regulations allow it, had signifi-

\begin{tabular}{|c|c|c|c|}
\hline Concern & $\begin{array}{l}\text { Mean value* } \\
\text { Academia }\end{array}$ & Nonacademia & $P$ (proportional odds) \\
\hline $\begin{array}{l}\text { There is no treatment site of }{ }^{131} \text {-tositumomab or } \\
90 \text {-ibritumomab tiuxetan convenient for my patients }\end{array}$ & 1.608 & 2.418 & $<0.01$ \\
\hline $\begin{array}{l}\text { It is or would be economically adverse for my practice to use } \\
{ }^{131} \text {-tositumomab or }{ }^{90} \text {-ibritumomab tiuxetan }\end{array}$ & 1.775 & 2.209 & $<0.01$ \\
\hline $\begin{array}{l}\text { The nuclear physicians are not too interested in treating patients with } \\
{ }_{131} \text {-tositumomab or }{ }^{90} \mathrm{Y} \text {-ibritumomab tiuxetan }\end{array}$ & 2.05 & 2.672 & $<0.01$ \\
\hline $\begin{array}{l}\text { Referring patients for }{ }^{131} \text {-tositumomab or }{ }^{90} Y \text {-ibritumomab tiuxetan } \\
\text { is too complicated }\end{array}$ & 2.138 & 2.724 & $<0.01$ \\
\hline $\begin{array}{l}{ }^{131} \text {-tositumomab or }{ }^{90} Y \text {-ibritumomab tiuxetan do not have enough } \\
\text { reported randomized studies }\end{array}$ & 2.45 & 2.343 & 0.8 \\
\hline${ }^{131}$-tositumomab or ${ }^{90} \mathrm{Y}$-ibritumomab tiuxetan is too expensive & 2.8 & 3.053 & 0.21 \\
\hline There are possible unexpected late side effects (MDS) & 2.938 & 3.104 & 0.14 \\
\hline $\begin{array}{l}\text { 131-tositumomab or }{ }^{90} \text {-ibritumomab tiuxetan theoretically may cause } \\
\text { bone marrow damage preventing further therapy (autologous stem } \\
\text { cell transplantation) }\end{array}$ & 2.988 & 3.269 & 0.08 \\
\hline $\begin{array}{l}\text { There are too many effective nonradioactive treatment alternatives } \\
\text { for the NHL }\end{array}$ & 3.013 & 2.993 & 0.98 \\
\hline
\end{tabular}


cantly more concerns about the lack of a treatment site $(P<0.01)$, the lack of interest by nuclear physicians $(P<$ $0.01)$, a referral process that was too complicated $(P<$ 0.01 ), and an adverse economic effect on their practice if they referred patients for radioimmunotherapy $(P<0.01)$.

Responders who thought radioimmunotherapy for NHL would probably or definitely grow in importance had significantly fewer concerns (or interests) than other responders about nonradioactive treatment alternatives $(P<0.01)$, bone marrow damage $(P<0.01)$, a perceived high price of ${ }^{90} \mathrm{Y}$-ibritumomab tiuxetan or ${ }^{131}$ I-tositumomab $(P=0.03)$, the lack of randomized trials $(P=0.01)$, and late side effects such as $\operatorname{MDS}(P=0.01)$.

Predicted Growth. Seventy-nine responders thought radioimmunotherapy with ${ }^{90}$ Y-ibritumomab tiuxetan or ${ }^{131}$ I-tositumomab would probably grow, and 52 responders thought it would definitely grow in importance. However, the optimistic responders could not be significantly assigned to a single practice type. The only additional significant factor by subgroup analysis was that responders who thought this treatment would grow saw greater growth if they could apply the treatment in their own offices $(P<$ $0.05)$.

\section{DISCUSSION}

${ }^{131}$ I-tositumomab and ${ }^{90}$ Y-ibritumomab tiuxetan radioimmunotherapy is FDA-approved for follicular and secondary transformed lymphoma after first-line therapy, and as of September 2009, ${ }^{90}$ Y-ibritumomab tiuxetan is FDAapproved as part of first-line therapy in both the United States and Europe. Despite the increasing evidence of efficacy and the relatively low reported toxicity, this relatively new form of treatment is not as extensively used as other therapies for lymphoma. This study is the first, to our knowledge, to analyze medical oncologists' perceptions and use of radioimmunotherapy with ${ }^{131}$ I-tositumomab or ${ }^{90}$ Y-ibritumomab tiuxetan in the United States. Our goal was to identify the challenges that need to be overcome to increase the use of radioimmunotherapy. Although new cancer therapies are typically more expensive than therapies using older drugs, determining how oncologists select therapies for their patients could add information to the health care debate.

Only $5.0 \%$ of the invited oncologists answered this survey. This number appears low but is in the expected range for e-mail-based surveys (11). Radioimmunotherapy is certainly used by our group of responders: 157 (72.7\%) had referred patients with NHL for radioimmunotherapy with ${ }^{131}$ I-tositumomab or ${ }^{90} \mathrm{Y}$-ibritumomab tiuxetan in the past 24 mo. However, there may be a bias because our responders may be more interested than the nonresponders in this form of treatment. More than $60 \%$ of the responders saw ${ }^{90}$ Y-ibritumomab tiuxetan or ${ }^{131}$ I-tositumomab as a suitable second- or first-line treatment for patients with NHL. An even a larger group saw radioimmunotherapy for later indications as third-line ( $n=138$ ) or even post-third-line therapy ( $n=$ 71 ), and their only significant concern was the availability of nonradioactive alternative treatments.

There are several agents for second-line treatment of FL. Retreatment with rituximab shows an overall response of $40 \%$, with $11 \%$ complete remissions (12). Rituximab, combined with cyclophosphamide, doxorubicin, vincristine, and prednisone, demonstrates a $30 \%$ complete response rate (13). Overall, there is a broad variety of choice in the treatment of lymphoma, and this might be one of the most important hurdles for the future of radioimmunotherapy $(14-18)$.

Fifty-four $(25 \%)$ responders would refer patients for radioimmunotherapy for consolidation after immunochemotherapy. This indication recently received regulatory approval in Europe and the United States for marketing. These responders did not significantly differ from the others in type of practice.

A recent randomized multicenter phase III trial (FIT Trial (6)) investigated ${ }^{90}$ Y-ibritumomab tiuxetan as firstline consolidation therapy. ${ }^{90}$ Y-ibritumomab tiuxetan was given as a single therapeutic dose in patients with advanced (stage III or IV) FL who achieved a partial or complete remission after receiving various induction chemotherapy regimens. In 414 patients, the results showed a significantly prolonged median progression-free survival, from 13.3 to 36.5 mo. However, in the small subgroup of patients receiving previous rituximab therapy, the prolongation of progression-free survival was not significant.

The nonradioactive alternative is prolonged rituximab maintenance. This treatment was recently analyzed in a large systematic review, and a higher rate of severe infections was observed. The result was a black-box warning issued by the FDA regarding long-term use of rituximab because of the occurrence of progressive multifocal leukoencephalopathy (19). However, superior rates of overall survival were found for patients with relapsed disease (hazard ratio, 0.58; confidence interval, 0.420.79), but the survival benefit was not consistent for patients after first-line treatment (20).

We observed the interplay of potential competing medical and economic interests in our survey. The responders who would consolidate had significantly fewer concerns about nonradioactive alternatives $(P=0.02)$ than those who would not consolidate, but these responders also perceived an adverse economic effect on their own practice $(P<0.01)$.

The future growth of radioimmunotherapy with ${ }^{131}$ I-tositumomab or ${ }^{90} \mathrm{Y}$-ibritumomab tiuxetan was judged to be positive by most of our responders. There was no single type of practice in which most of the responders had a positive outlook about the future growth of radioimmunotherapy. However, logistic regression did show that positive responders thought radioimmunotherapy for NHL would grow even more if it could be given in their own practice $(P=$ 0.03). Responders who thought that referring patients away 
from their practice for treatment would be economically adverse to their practice also thought radioimmunotherapy would grow more if it could be given in their own practice $(P<0.01)$. This response clearly showed a high economic stake affecting perceptions about radioimmunotherapy use, which is a factor in the perception of growth for the future of radioimmunotherapy therapy.

In the free-text field a responder wrote, "These drugs are of great value in patients with lymphoma but will never be used in the number of patients that could benefit because of present logistic and regulatory restrictions. If the drug could be easily ordered or even administered by medical oncologists, this would improve the situation greatly." The effects that beneficiaries and generous reimbursement have on the choice of cancer treatment have been discussed previously $(21,22)$.

Responders not from academia were significantly more concerned than those from academia that referral of patients for radioimmunotherapy would have an adverse economic effect on their practice $(P<0.01)$. One responder wrote, "This treatment is underutilized and difficult to arrange at tertiary centers, I believe, in large part because of the reimbursement reward to practicing oncologists and the need to involve and educate nuclear medicine physicians." This comment and others clearly demonstrate the importance of logistic and economic factors affecting the availability of radioimmunotherapy for NHL.

The nonacademic responders were significantly more concerned about the lack of a treatment site, the lack of interest by nuclear physicians, and a referral process that was too complicated.

The only significant concern in the group that did not treat any NHL patients within the past 24 mo was the high price of ${ }^{131}$ I-tositumomab and ${ }^{90}$ Y-ibritumomab tiuxetan. Currently, Medicare reimburses hospitals about $\$ 16,000$ for each treatment with radioimmunotherapy (23). The cost for combined chemoimmunotherapy with cyclophosphamide, doxorubicin, vincristine, and prednisone and rituximab is about $\$ 17,000$ (24), making the price for these second-line treatments comparable. The reimbursement practice by Medicare for ${ }^{90}$ Y-ibritumomab tiuxetan and ${ }^{131}$ I-tositumomab has been questioned, and hospitals administering radioimmunotherapy to patients with NHL may lose about $\$ 10,000$ per treatment (25). This might be one of several explanations of why some hospitals stopped providing radioimmunotherapy for patients with NHL.

\section{CONCLUSION}

Overall, we see a positive perception for radioimmunotherapy in this population of referring U.S. oncologists. Our data indicate that economic and logistic effects on the oncologists' practice are important. Some fear that by referring a patient away from their practice for radioimmunotherapy, they may lose that patient. Others fear the loss of revenue from treatments at their own site resulting from referral to other sites for this treatment. This could be one of several reasons why radioimmunotherapy is not more widely used. Of course, in an ideal world, the choice of cancer treatment for a specific patient should be based on the most effective therapy for that patient, and perhaps on its cost-effectiveness, without any influence from its profitability to the treating physician.

It seems crucial for the future of radioimmunotherapy not only to demonstrate that the treatment process is safe and efficacious but also to streamline the process to provide easy referrals and better collaboration among specialists and to ensure that appropriate safeguards are developed so that the economic concerns of the referring physician are not a significant factor in their choice of cancer treatment for patients with lymphoma.

\section{ACKNOWLEDGMENTS}

We thank Dr. Richard F. Ambinder for his kind support. Dr. Richard L. Wahl is one of the inventors of anti-CD20 radioimmunotherapy and receives payments from the University of Michigan as part of a patent licensing agreement related to royalties from the U.S. sales of the radioimmunotherapy agents ${ }^{131}$ I-tositumomab and ${ }^{90}$ Y-ibritumomab tiuxetan. This arrangement has been disclosed to and is managed by the JHU COI committee.

\section{REFERENCES}

1. Kaminski MS, Zasadny KR, Francis IR, et al. Radioimmunotherapy of B-cell lymphoma with [ ${ }^{131} \mathrm{I}$ ] anti-B1 (anti-CD20) antibody. N Engl J Med. 1993;329: 459-465.

2. Vose JM, Wahl RL, Saleh M, et al. Multicenter phase II study of iodine-131 tositumomab for chemotherapy-relapsed/refractory low-grade and transformed low-grade B-cell non-Hodgkin's lymphomas. J Clin Oncol. 2000;18:1316-1323.

3. Kaminski MS, Tuck M, Estes J, et al. ${ }^{131} \mathrm{I}$-tositumomab therapy as initial treatment for follicular lymphoma. N Engl J Med. 2005;352:441-449.

4. Witzig TE, Gordon LI, Cabanillas F, et al. Randomized controlled trial of yttrium-90-labeled ibritumomab tiuxetan radioimmunotherapy versus rituximab immunotherapy for patients with relapsed or refractory low-grade, follicular, or transformed B-cell non-Hodgkin's lymphoma. J Clin Oncol. 2002;20:24532463.

5. Wiseman GA, Gordon LI, Multani PS, et al. Ibritumomab tiuxetan radioimmunotherapy for patients with relapsed or refractory non-Hodgkin lymphoma and mild thrombocytopenia: a phase II multicenter trial. Blood. 2002;99:43364342 .

6. Witzig TE, Flinn IW, Gordon LI, et al. Treatment with ibritumomab tiuxetan radioimmunotherapy in patients with rituximab-refractory follicular nonHodgkin's lymphoma. J Clin Oncol. 2002;22:3262-3269.

7. Morschhauser F, Radford J, Van Hoof A, et al. Phase III trial of consolidation therapy with yttrium-90-ibritumomab tiuxetan compared with no additional therapy after first remission in advanced follicular lymphoma. J Clin Oncol. 2008;26:5156-5164.

8. Leahy MF, Seymour JF, Hicks RJ, Turner JH. Multicenter phase II clinical study of iodine-131-rituximab radioimmunotherapy in relapsed or refractory indolent non-Hodgkin's lymphoma. J Clin Oncol. 2006;24:4418-4425.

9. Berenson A. Market forces cited in lymphoma drugs' disuse. New York Times. July 14, 2007. Available at: http://www.nytimes.com/2007/07/14/health/14lymphoma. html. Accessed April 20, 2010.

10. Otte A. Does health economics have an impact on non-Hodgkin's lymphoma patients' options? Nucl Med Commun. 2008;29:748-749.

11. Hewson C, Yule P, Laurent D, Vogel C. Internet Research Methods: A Practical Guide for the Social and Behavioural Sciences. London, U.K.: Sage Publications Ltd.; 2003. 
12. Davis TA, Grillo-Lopez AJ, White CA, et al. Rituximab anti-CD20 monoclonal antibody therapy in non-Hodgkin's lymphoma: safety and efficacy of re-treatment. J Clin Oncol. 2000;18:3135-3143.

13. van Oers MH, Klasa R, Marcus RE, et al. Rituximab maintenance improves clinical outcome of relapsed/resistant follicular non-Hodgkin lymphoma in patients both with and without rituximab during induction: results of a prospective randomized phase 3 intergroup trial. Blood. 2006;108:3295-3301.

14. Robinson KS, Williams ME, van der Jagt RH, et al. Phase II multicenter study of bendamustine plus rituximab in patients with relapsed indolent B-cell and mantle cell non-Hodgkin's lymphoma. J Clin Oncol. 2008;26:4473-4479.

15. Friedberg JW, Cohen P, Chen L, et al. Bendamustine in patients with rituximabrefractory indolent and transformed non-Hodgkin's lymphoma: results from a phase II multicenter, single-agent study. J Clin Oncol. 2008;26:204-210.

16. Forstpointner R, Dreyling M, Repp R, et al. The addition of rituximab to a combination of fludarabine, cyclophosphamide, mitoxantrone (FCM) significantly increases the response rate and prolongs survival as compared with FCM alone in patients with relapsed and refractory follicular and mantle cell lymphomas: results of a prospective randomized study of the German LowGrade Lymphoma Study Group. Blood. 2004;104:3064-3071.

17. Sacchi S, Pozzi S, Marcheselli R, et al. Rituximab in combination with fludarabine and cyclophosphamide in the treatment of patients with recurrent follicular lymphoma. Cancer. 2007;110:121-128.
18. Jacene HA, Filice R, Kasecamp W, Wahl RL. Comparison of ${ }^{90}$ Y-ibritumomab tiuxetan and ${ }^{131}$ I-tositumomab in clinical practice. J Nucl Med. 2007;48:17671776.

19. Information for Healthcare Professionals: Rituximab (marketed as Rituxan). Available at: www.fda.gov/Drugs/DrugSafety/PostmarketDrugSafetyInformation forPatientsandProviders/ucm126519.htm. Accessed April 20, 2010.

20. Vidal L, Gafter-Gvili A, Leibovici L, et al. Rituximab maintenance for the treatment of patients with follicular lymphoma: systematic review and metaanalysis of randomized trials. J Natl Cancer Inst. 2009;101:248-255.

21. Iglehart JK. Medicare and drug pricing. N Engl J Med. 2003;348:1590-1597.

22. Jacobson M, O'Malley AJ, Earle CC, Pakes J, Gaccione P, Newhouse JP. Does reimbursement influence chemotherapy treatment for cancer patients? Health Aff (Millwood). 2006;25:437-443.

23. Centers for Medicare and Medicaid Services, Department of Health and Human Services. Available at: http://www.cms.hhs.gov/. Accessed April 20, 2010.

24. Hornberger JC, Best JH. Cost utility in the United States of rituximab plus cyclophosphamide, doxorubicin, vincristine, and prednisone for the treatment of elderly patients with diffuse large B-cell lymphoma. Cancer. 2005;103:16441651.

25. Berenson A. Medicare cuts payout on 2 cancer drugs. New York Times. December 7, 2007. Available at: http://www.nytimes.com/2007/12/07/business/ 07drug.html. Accessed April 20, 2010. 